\title{
Revenue Monotone Mechanisms for Online Advertising
}

\author{
Gagan Goel \\ Google Inc., 76 Ninth Ave \\ New York, NY 10011 \\ gagangoel@google.com
}

\author{
Mohammad Reza Khani ${ }^{*}$ \\ University of Maryland \\ College Park, MD 20740 \\ khani@cs.umd.edu
}

\begin{abstract}
Online advertising is an essential part of the Internet and the main source of revenue for many web-centric firms such as search engines, social networks, and online publishers. A key component of online advertising is the auction mechanism which selects and prices the set of winning ads.

This work is inspired by one of the biggest practical drawbacks of the widely popular Vickrey-Clarke-Groves (VCG) mechanism, which is the unique incentive-compatible mechanism that maximizes social welfare. It is known that VCG lacks a desired property of revenue monotonicity - a natural notion which states that the revenue of a mechanism shouldn't go down as the number of bidders increase or if the bidders increase their bids. Most firms which depend on online advertising revenue have a large sales team to attract more bidders on their inventory as the general belief is that more bidders will increase competition, and hence revenue. However, the lack of revenue monotonicity of VCG conflicts with this general belief and can be strategically confusing for the firm's business.

In this work, we seek incentive-compatible mechanisms that are revenue-monotone. This natural property comes at the expense of social welfare - one can show that it is not possible to get incentive-compatibility, revenue-monotonicity, and optimal social welfare simultaneously. In light of this, we introduce the notion of Price of Revenue Monotonicity (PoRM) to capture the loss in social welfare of a revenuemonotone mechanism.

We further study revenue-monotonicity for two important online advertising scenarios. First one is the text vs image ad auction where in an ad slot, one can either show a single image ad or a few text ads. Second one is the video-pod auction where we have a video advertising slot of $k$ seconds

\footnotetext{
*This work was done while the second author was an intern at Google, New York, and student at University of Maryland, College park where supported in part by NSF CAREER award 1053605, NSF grant CCF-1161626, ONR YIP award N000141110662, DARPA/AFOSR grant FA9550-12$1-0423$
}

Copyright is held by the International World Wide Web Conference Committee (IW3C2). IW3C2 reserves the right to provide a hyperlink to the author's site if the Material is used in electronic media. WWW'14, April 7-11, 2014, Seoul, Korea. ACM 978-1-4503-2744-2/14/04.

http://dx.doi.org/10.1145/2566486.2568000. which can be filled with multiple video ads. For the imagetext auction, we give a mechanism that satisfy both RM and IC and achieve PoRM of $\sum_{i=1}^{k} \frac{1}{i} \approx \ln k$. We also show that the PoRM of our mechanism is the best possible by proving a matching lower bound of $\sum_{i=1}^{k} \frac{1}{i}$ on the PoRM of any deterministic mechanism under some mild assumptions. For the video-pod auction, we give a mechanism that achieves a PoRM of $(\lfloor\log k\rfloor+1) \cdot(2+\ln k)$.

\section{Categories and Subject Descriptors}

K.4.4 [Computers and Society]: Electronic Commerce Payment Schemes

\section{General Terms}

Algorithmic Game Theory, Mechanism Design

\section{Keywords}

Incentive Compatibility, Revenue Monotonicity, Social Welfare, Approximation, Price of Revenue Monotonicity

\section{INTRODUCTION}

Fueled by the growth of Internet and advancements in online advertising techniques, today more and more online firms rely on advertising revenue for their business. Some of these firms include news agencies, media outlets, search engines, social and professional networks, etc. Much of this online advertising business is moving to what's called programmatic buying where an advertiser bids for each single impression, sometimes in real-time, depending on how he values the ad opportunity. This work is motivated by the need of a desired property in the auction mechanisms that are used in these bid-based advertising systems.

A standard mechanism for most auction scenarios is the famous Vickrey-Clarke-Groves (VCG) mechanism. VCG is incentive-compatible (IC) and maximizes social welfare. Incentive-compatibility guarantees that the best response for each advertiser is to report its true valuation. This makes the mechanism transparent and removes the load from the advertisers to calculate the best response. Social welfare is the sum of the valuations of the winners. This value is treated as a proxy for how much all the participants gain from the transaction. What makes VCG mechanism versatile is that it reduces the mechanism design problem into an optimization problem for any scenario.

Even though this versatility of VCG mechanism makes it a popular choice mechanism, however, it doesn't satisfy an 
important property, namely, that of revenue-monotonicity. Revenue-monotonicity says that if one increases the bid values or add new bidders, the total revenue should not go down. To see that VCG is not revenue-monotone, consider a simple example of two items and three bidders (A, B, and C). Say bidder A wants only the first item, and has a bid of 2. Similarly bidder B wants only the second item, and has a bid of 2. Bidder $\mathrm{C}$ wants both the items or nothing, and has a bid of 2. Now if only bidders A and B participate in the auction, then VCG gives a revenue of 2 , however, if all the three bidders participate, then the revenue goes down to 0 .

This lack of revenue-monotonicity (which has been noted several times in the literature) is one of the serious practical drawbacks of the celebrated VCG mechanism. To think of it, an online firm that depends on advertising revenue puts significant resources in its sales efforts to attract more bidders as the general belief is that more bidders imply more competition which should lead to higher prices. Now to tell this firm that their revenue can go down if they get more bidders can be strategically very confusing for them. To see this from another perspective, say in a search engine firm, there is a team which makes a UI change that increases the click-through probability (CTR) of the search ads. These changes are thought of as good changes in the firm as they increase the effective bid of the bidders (the effective bid of a bidder in search advertising is a function of its cost-perclick bid and the CTR of its ad). Now if after making the change, the revenue goes down, what was supposed to be a good change may seem like a bad change. The point we are trying to make is that there are many teams in a firm, and for these teams to function properly, it is important that the auction mechanisms satisfy revenue-monotonicity.

In this paper, with a focus on auctions arising in advertising scenarios, we seek to understand mechanisms that satisfy this additional property of revenue monotonicity (RM). It is well known that for various settings (including ours), no mechanism can satisfy both IC and RM properties while attaining optimal social welfare. In fact it is known that one cannot even hope to get Pareto-optimality in social welfare while attaining both IC and RM [10]. Thus to overcome this bottleneck and develop an understanding of RM mechanisms, we relax the requirement of attaining full social welfare, and define the notion of price of revenue-monotonicity (PoRM). Price of revenue-monotonicity of an IC and RM mechanism $\mathrm{M}$ is the ratio of optimal social welfare to the social welfare attained by the mechanism M. The goal is to design mechanisms that satisfy IC and RM properties and at the same time achieve low price of revenue-monotonicity. To the best of our knowledge, this is the first work that defines and studies this notion of price of revenue-monotonicity.

We study two different advertising settings in this paper. The first setting we study is the image-text auction. In image-text auction there is a special box designated for advertising in a publisher's website which can be filled by either $k$ text-ads or a single image-ad. The second setting is the video-pod auction where an advertising break of a certain duration in a video content can be filled with multiple video ads of possibly different durations.

We note that revenue-monotonicity is an across-instance constraint as it requires total revenue to behave in a certain manner across different instances, where a single instance is defined by fixing the type of the buyers. Note that incentive-compatibility is also an across-instance con- straint. A lot of research effort has gone into understanding incentive-compatibility, which has resulted in useful tools for designing incentive-compatible mechanisms. Surprisingly, hardly any work has gone into understanding and building tools for designing mechanisms which satisfy the desired property of revenue-monotonicity. We believe that understanding revenue-monotonicity will shed new fundamental insights into the design of mechanisms for many practical scenarios.

\subsection{Related Work}

Ausubel and Milgrom [1] show that VCG satisfies RM if bidders' valuations satisfy bidder-submodularity. Bidders' valuations satisfy bidders submodularity if and only if for any bidder $i$ and any two sets of bidders $S, S^{\prime}$ with $S \subseteq S^{\prime}$ we have $\mathrm{WF}(S \cup\{i\})-\mathrm{WF}(S) \geq \mathrm{WF}\left(S^{\prime} \cup\{i\}\right)-\mathrm{WF}\left(S^{\prime}\right)$, where $\mathrm{WF}(S)$ is the maximum social welfare achievable using only $S$. Note that this is a general tool one can use to design revenue monotone mechanisms - restrict the range of the possible allocations such that we get bidder-submodularity when we run VCG on this range. However, we can show that this general tool is not so powerful by showing that for our auction scenarios, it is not possible to get a mechanism with PoRM better than $\Omega(k)$ by using the above tool.

Ausubel and Milgrom [1] also show that biddersubmodularity is guaranteed when the goods are substitutes, i.e., the valuation function of each bidder is submodular over the goods. However, for many practical scenarios, including ours, the valuation function of the bidders is not submodular. Ausubel and Milgrom [1] design mechanisms which select allocations that are in the core of the exchange economy for combinatorial auctions. Here an allocation is in the core if there is no coalition of bidders and the seller to trade with each other in a way which is preferred by all the members of the coalition to the allocation. Day and Milgrom [3] show that core-selecting mechanisms that choose a core allocation which minimizes the seller's revenue satisfy RM given bidders follow so called best-response truncation strategy. Therefore the core selecting mechanism designed by [1] satisfies RM if the participants play such best-response strategy; although this mechanism is not incentive-compatible.

Rastegari et al. [10] prove that no mechanism for general combinatorial auctions which satisfies IC and RM can achieve weakly maximal social welfare. An allocation is weakly maximal if it cannot be modified to make at least one participant better off without hurting anyone else. In another work [9] they design a randomized mechanism for combinatorial auctions which achieves weak maximality and expected revenue monotonicity.

Another related work is around the characterization of mechanisms that achieve the IC property. The classic result of Roberts [11] states that affine maximizers are the only social choice functions that can be implemented using IC mechanisms when bidders have unrestricted quasilinear valuations. Subsequent works study the restricted cases $[2,6,12,13]$.

There is also an extensive body of research around designing mechanisms with good bounds on the revenue. Myerson [7] designs a mechanism which achieves the optimal expected revenue in the single parameter Bayesian setting. Goldbert et al. consider optimizing revenue in prior-free settings (see [8] for a survey on this). 


\subsection{Our Results}

As mentioned earlier, we study two settings: 1) imagetext auction, and 2) video-pod auction. Both these settings can be described using the following abstract model. Say there is a seller selling $k$ identical items to $n$ participants/buyers. Participant $i$ wants either $d_{i}$ items or nothing, and has a valuation of $v_{i}$ if gets $d_{i}$ items or 0 otherwise. Demand $d_{i}$ is assumed to be public knowledge, and valuation $v_{i}$ is assumed to be the private information of the participant $i$. We want to design a mechanism that is incentive-compatible, individually-rational (IR), revenuemonotone, and maximizes social welfare.

For the image-text auction, the demand $d_{i} \in\{1, k\}$, i.e. each participant wants either 1 item (text ads) or $\mathrm{k}$ items (image ad). For the video-pod auction, an item corresponds to a unit time interval (say one second), and the demand $d_{i}$ could be any number between 1 and $k$, i.e., $d_{i} \in[k]$.

The first result of this paper is the following theorem.

Theorem 1 We design a deterministic mechanism for image-text auction (MITA) which satisfies Individual Rationality (IR), IC, and RM with PoRM of at most $\sum_{i=1}^{k} \frac{1}{i} \simeq$ $\ln (k)$, i.e., the ratio of MITA's welfare over the optimal welfare is at most $\ln (k)$.

The proof of Theorem 1 appears in Section 3. We outline our mechanism over here: Let $v_{1} \geq \ldots \geq v_{n_{1}}$ be the valuations of text-participants and $V_{1}$ be the maximum valuation of the image-participants. If $\max _{j \in[k]} j \cdot v_{j}$ is less than $V_{1}$, MITA gives all the items to the image-participant who has valuation $V_{1}$, otherwise MITA picks the highest $j^{*}$ text-participants as the winners where $j^{*}$ is the maximum number in $[k]$ such that $j^{*} \cdot v_{j^{*}} \geq V_{1}$. Note that the $j$ that maximizes $j \cdot v_{j}$ might be less than the $j *$ which is the largest $j$ such that $j \cdot v_{j} \geq V_{1}$. Also note that MITA sometimes picks less than $k$ text ads as the winner (even if there are $k$ or more text ads). VCG always picks the maximum number of text ads (if it decides to allocate the slot to text ads); this is one of the reasons why VCG fails to satisfy RM. When we allow lesser number of text ads to be declared as winners, intuitively, this increases the competition which boosts the revenue and thus helps in achieving RM. Although this comes with a loss in social welfare.

Surprisingly, we can also show that the above mechanism achieves the optimal PoRM for the image-text auction by proving a matching lower bound. We show that a mechanism that satisfies IR, IC, RM, and two additional mild assumptions of Anonymity (AM) and Independence of Irrelevant Alternatives (IIA) cannot achieve a PoRM better than $\sum_{i=1}^{k} \frac{1}{i}$. Anonymity means that the auction mechanism doesn't depend on the identities of the participants (a formal definition appears in Section 5). IIA means that decreasing the bid of a loosing participant shouldn't hurt any winner. Note that our mechanism satisfy both AM and IIA as well. Formally, we prove the following theorem whose proof appears in Section 5.

Theorem 2 There is no deterministic mechanism which satisfies IR, IC, RM, AM, and IIA and has PoRM less than $\sum_{i=1}^{k} \frac{1}{i}$.

Finally we prove the following theorem for video-pod auctions.
Theorem 3 We design a Mechanism for Video-pod Auction (MVPA) which satisfies IR, IC, and RM with PoRM of at most $(\lfloor\log k\rfloor+1) \cdot(2+\ln k)$.

We give the formal proof of Theorem 3 in Section 4, and outline the mechanism here. MVPA partitions the participants into $(\lfloor\log k\rfloor+1)$ groups where each group $g \in[\log k]$ contains only the participants whose demands are in the range $\left[2^{g-1}, 2^{g}\right)$. MVPA selects winners only from one group. We round up the size of each participant in group $g$ to $2^{g}$, thus we can have at most $\frac{k}{2^{g}}$ number of winners from the group g. Let $v_{1}^{(g)} \geq \ldots \geq v_{p}^{(g)}$ be the sorted valuations of all the participants in group $g$. We define the Max Possible Revenue of Group $g$ (MPRG $(g))$ to be

$$
\operatorname{MPRG}(g)=\max _{j \in\left[k / 2^{g}\right]} j \cdot v_{j}^{(g)} .
$$

As the name of $\operatorname{MPRG}(g)$ suggests, its value captures the maximum revenue we can truthfully obtain from group $g$ without violating revenue-monotonicity. Let $g^{*}$ be the group with the highest MPRG value and group $g^{\prime}$ be the group whose MPRG is second highest. The set of winners are the first $j$ participants from group $g^{*}$ where $j$ is the largest number in $\left[k / 2^{g}\right]$ such that $j \cdot v_{j}^{\left(g^{*}\right)}$ is greater than or equal MPRG $\left(g^{\prime}\right)$. We show that PORM of MVPA is $(\lfloor\log k\rfloor+1) \cdot(2+\ln k)$.

\section{PRELIMINARIES}

Let $N=\{1, \ldots, n\}$ be the set of all participants, and $k$ be the number of identical items. We denote the type of participant $i$ by $\theta_{i}=\left(d_{i}, v_{i}\right) \in[k] \times \mathbb{R}^{+}$, where $d_{i}$ is the number of items participant $i$ demands and $v_{i}$ is her valuation for getting $d_{i}$ items. Note that the valuation of player $i$ for getting less than $d_{i}$ items is 0 . Now in the image-text auction, participants have demand of either 1 or $k$. In the video-pod auction participants can have arbitrary demands in $\{1, \ldots, k\}$. Lets denote the set of all possible types $[k] \times \mathbb{R}^{+}$by $\Theta$ and the set of all type profiles of $n$ participants by $\Theta^{n}=\underbrace{\Theta \times \ldots \times \Theta}_{n}$.

A deterministic mechanism $\mathcal{M}$ consists of an allocation rule $x: \Theta^{n} \rightarrow 2^{n}$ which maps each type profile to a subset of participants as the winners, and payment rule $p: \Theta^{n} \rightarrow$ $\left(\mathbb{R}^{+}\right)^{n}$ which maps each type profile to the payments of each participant.

Let $\theta=\left(\theta_{1}, \theta_{2}, \ldots, \theta_{n}\right) \in \Theta^{n}$ be a specific type profile. Also let $\mathcal{A}_{\theta}$ be the set of all feasible solutions, i.e.,

$$
\mathcal{A}_{\theta}=\left\{S \subseteq N \mid \sum_{i \in S} d_{i} \leq k\right\} .
$$

For each feasible solution $A \in \mathcal{A}_{\theta}$, the social welfare of $A$ (denoted by $\mathrm{WF}(A))$ is equal to $\sum_{\theta_{i} \in A} v_{i}$. To evaluate the social welfare of a mechanism $\mathcal{M}$ on a type profile $\theta$, we compare the welfare of its solution to the optimal solution.

Definition 1 The welfare ratio of mechanism $\mathcal{M}=(x, p)$ on type profile $\theta \in \Theta^{n}$ (denoted by $\operatorname{WFR}(\mathcal{M}, \theta)$ ) is the following.

$$
\operatorname{WFR}(\mathcal{M}, \theta)=\frac{\max _{A \in \mathcal{A}_{\theta}} \mathrm{WF}(A)}{\mathrm{WF}(x(\theta))}
$$


To capture the worst-case loss in social welfare across all type profiles, we define the notion of price of revenuemonotonicity.

Definition 2 The Price of Revenue Monotonicity of a mechanism $\mathcal{M}$ (denoted by $\operatorname{PoRM}(\mathcal{M})$ ) is defined as follows:

$$
\operatorname{PoRM}(\mathcal{M})=\max _{\theta \in \Theta^{n}} \operatorname{WFR}(\mathcal{M}, \theta)
$$

The desired goal is to design mechanisms which have low PoRM value, where the best possible value is 1 .

Note that since we are interested in mechanisms with bounded PoRM, we restrict ourselves to mechanisms that satisfy consumer sovereignty. Consumer sovereignty says that any participant can be a winner as long as he bids high enough.

Now we will define a weakly monotone allocation rule which is used in the characterization of deterministic IC mechanisms. Let function $x_{i}: \Theta^{n} \rightarrow\{0,1\}$ be the restriction of function $x$ to participant $i$. Here $x_{i}($.$) is one$ if participant $i$ is a winner and zero otherwise.

Definition 3 We call allocation function $x$ is weakly monotone if for any type profile $\theta \in \Theta^{n}$ and any participant $i \in[n]$ with demand $d_{i}$, function $x_{i}\left(\left(d_{i}, v_{i}\right), \theta_{-i}\right)$ is a nondecreasing function in $v_{i}$.

Note that if a deterministic mechanism $\mathcal{M}$ satisfies consumer sovereignty and has a weakly monotone allocation function then function $x_{i}\left(\left(d_{i}, v_{i}\right), \theta_{-i}\right)$ is a single step function. The value at which the function $x_{i}\left(\left(d_{i}, v_{i}\right), \theta_{-i}\right)$ jumps from zero to one, i.e. the smallest value at which the participant $i$ becomes a winner, is called critical value.

Definition 4 Let $\mathcal{M}=(x, p)$ be a deterministic mechanism that satisfy consumer sovereignty and has a weakly monotone allocation function, the critical value of participant $i$ in type profile $\theta$ is $v_{i}^{*}=\sup \left\{v_{i} \mid x_{i}\left(\left(d_{i}, v_{i}\right), \theta_{-i}\right)=0\right\}$.

The following lemma characterizes deterministic IC mechanisms (first given by [7]). We provide a proof sketch for the sake of completeness (for a complete proof see e.g. [8]).

Lemma 1 Let $\mathcal{M}=(x, p)$ be a mechanism which satisfies IR. Mechanism $\mathcal{M}$ is truthful (IC) if and only if the followings hold.

\section{1. $x$ is weakly monotone.}

2. If participant $i$ is a winner then its payment is its critical value $\left(v_{i}^{*}\right)$.

Proof. First we prove that if $\mathcal{M}$ is truthful then it satisfies both conditions 1 and 2 . We prove the first condition by contradiction. If $x$ is not monotone then there exist participant $i$, type profile $\theta$, and two values $v_{i}^{(1)}>v_{i}^{(2)}$ such that $i$ wins in type profile $\left(\left(d_{i}, v_{i}^{(2)}\right), \theta_{-i}\right)$ but loses in type profile $\left(\left(d_{i}, v_{i}^{(1)}\right), \theta_{-i}\right)$. This makes incentive for participant $i$ to lie for type profile $\left(\left(d_{i}, v_{i}^{(1)}\right), \theta_{-i}\right)$ and announce its valuation as $v_{i}^{(2)}$.

Consider an arbitrary participant $i$ who is a winner, now we prove that the payment of participant $i$ is its critical value. Assume for contradiction that mechanism $\mathcal{M}$ charges participant $i$ amount $c_{i}$ where $c_{i}<v_{i}^{*}$ in a type profile $\left(\left(d_{i}, v_{i}\right), \theta_{-i}\right)$. In this case, if participant $i$ had type $\left(d_{i}, \hat{v}_{i}\right)$ where $c_{i}<\hat{v}_{i}<v_{i}^{*}$ then $i$ is not a winner in $\left(\left(d_{i}, \hat{v}_{i}\right), \theta_{-i}\right)$ as $v_{i}^{*}$ is the critical value. Therefore, if the real type of participant $i$ is $\left(d_{i}, \hat{v}_{i}\right)$, she has incentive to lie her type as $\left(d_{i}, v_{i}\right)$, become a winner, and pay $c_{i}$. Hence, the payment cannot be less than $v_{i}^{*}$. Now suppose that there exists value $v_{i}$ for which mechanism $\mathcal{M}$ charges $i$ amount $c_{i}$ which is more than $v_{i}^{*}$. In this case, if participant $i$ had type $\left(d_{i}, \hat{v}_{i}\right)$ where $v_{i}^{*}<\hat{v}_{i}<c_{i}$ then $i$ is still a winner (as $v_{i}^{*}$ is the critical value) and pays at most $\hat{v}_{i}$ (as $\mathcal{M}$ satisfies IR). Therefore, she has an incentive to lie her type as $\left(d_{i}, \hat{v}_{i}\right)$, become a winner, and pay at most $\hat{v}_{i}$. Hence, the payment cannot be more than $v_{i}^{*}$ for any winning valuation $v_{i}$.

For the other direction, it is easy to check that any IR mechanism that satisfies conditions 1 and 2 is truthful.

\section{IMAGE-TEXT AUCTIONS}

In this section we give our Mechanism for ImageText Auction (MITA) which satisfies IR, IC, RM, and $\operatorname{PoRM}(\mathrm{MITA}) \leq \ln k$. Recall that in the image-text auction we have $k$ identical items to sell and there are two groups of participants: the ones who want all the $k$ items which we call image-participants; and the ones who want only one item which we refer to as text-participants. As a result there are also two possible types of outcome: MITA gives all the items to an image-participant; or it gives an item to each member of a subset of the text-participants.

We start with explaining why VCG fails to satisfy RM and how we address this issue in MITA. Consider the type profile where we have one image-participant with type $(k, 1)$ and one text-participant with type $(1,1)$. In this case either of the participants can be the winner. The payment of the winner in VCG is her critical value which is one. However if we add one more text-participant with the same type $(1,1)$, the two text-participants win and each of them pay zero. The reason for the payment drop is that VCG always selects $k$ winners from the text-participants. This decreases the critical value of each text participant as the valuation of the other text-participants helps her to win against image-participants. In our mechanism we overcome this issue by not guaranteeing that the maximal number of text-participants can win an item. In other words, in our mechanism it is possible that less than $k$ text-participants win an item even if there are more than $k$ text-participants. This way, intuitively, even if the number of text-participants increase, it potentially creates more competition and hence increases the payments.

Let $\theta$ be an arbitrary type profile where there are $n_{1}$ textparticipants with types $\left(1, v_{1}\right), \ldots,\left(1, v_{n_{1}}\right)$ and $n_{2}$ imageparticipants with types $\left(k, V_{1}\right), \ldots,\left(k, V_{n_{2}}\right)$. We define mechanism MITA $=\left(x^{\text {MITA }}, p^{\text {MITA }}\right)$ by giving allocation function $x^{\text {MITA }}$ which is weakly monotone. Given the allocation function, we obtain payment function $p^{\text {MITA }}$ using the critical values defined in Lemma 1 which makes the mechanism truthful.

Allocation rule of MITA. Without loss of generality we assume that $v_{1} \geq v_{2} \geq \ldots \geq v_{n_{1}}$ and $V_{1} \geq V_{2} \geq \ldots \geq V_{n_{2}}$. Also, we assume that $n_{1} \geq k$, if not, we add fake textparticipants with value 0 . For each $j \in[k]$ we consider value $j \cdot v_{j}$. Let candidate set $C_{\theta}$ contains all the values 
$j \in[k]$ such that $j \cdot v_{j}$ is greater than or equal to $V_{1}$, i.e., $C_{\theta}=\left\{j \in[k] \mid j \cdot v_{j} \geq V_{1}\right\}$. If $C_{\theta}$ is empty, the imageparticipant with type $\left(k, V_{1}\right)$ wins. If $C_{\theta}$ is non-empty then let $j^{*}$ be the maximum member of $C_{\theta}$, i.e., $j^{*}=\max _{j \in C_{\theta}} j$. In this case the first $j^{*}$ text-participants win.

\section{Observation 1 Allocation function $x^{\mathrm{MITA}}$ is weakly mono-} tone.

Proof. Recall from Definition 3, in order to prove that $x^{\text {MITA }}$ is weakly monotone, we have to show that for any participant $i \in[n]$ with demand $d_{i}$, function $x_{i}\left(\left(d_{i}, v_{i}\right), \theta_{-i}\right)$ is a non-decreasing function in $v_{i}$.

If $i$ is an image-participant then $i$ wins if its valuation is larger than $\max \left(W, \max _{j \in[k]} j \cdot v_{j}\right)$ where $W$ is the largest valuation of the image-participants in $\theta_{-i}$. Moreover, bidder $i$ loses for any value smaller than or equal to $\max \left(W, \max _{j \in[k]} j \cdot v_{j}\right)$. Therefore $x_{i}$ is weakly monotone.

If $i$ is a text-participant then let $v_{1}^{\prime} \geq v_{2}^{\prime} \geq \ldots$ be the sorted valuations of the text-participants and $V_{1}$ be the largest valuation of image-participants in $\theta_{-i}$. Let $t$ be the smallest value such that there exist $j \in[k-1]$ where $v_{j+1}^{\prime} \leq t \leq v_{j}^{\prime}$ and $(j+1) \cdot t$ is greater than or equal to $V_{1}$. If the valuation of bidder $i$ is larger than or equal to $t$ then she wins since $(j+1) \cdot t \geq V_{1}$ otherwise she does not win since $t$ is the smallest value for which there exist $j \in[k-1]$ such that $(j+1) \cdot t \geq V_{1}$. Therefore $x_{i}$ is weakly monotone.

In the following lemma we obtain the critical value (or truthful payments) of the winners in $x^{\text {MITA }}$ using Lemma 1. The lemma also gives an intuition to why we select $j^{*}$ textparticipants to win, which is the maximum $j$ such that $j$. $v_{j} \geq V_{1}$.

Lemma 2 If $C_{\theta}$, where $C_{\theta}=\left\{j \in[k] \mid j \cdot v_{j} \geq V_{1}\right\}$, is empty then the first image-participant wins all the items with critical value $\max \left(V_{2}, \max _{j \in[k]} j \cdot v_{j}\right)$. If $C_{\theta}$ is not empty, the first $j^{*}$ text-participants win the items where $j^{*}=\max _{j \in C_{\theta}} j$ and all of them have critical value $\max \left(v_{k+1}, \frac{V_{1}}{j^{*}}\right)$.

Proof. We find the critical value (Definition 4) of a winner by showing that if she has any valuation larger than the critical value she wins and for any valuation less than the critical value she doesn't.

If $C_{\theta}$ is empty then the first image-participant (with type $\left.\left(k, V_{1}\right)\right)$ wins all the items. As long as $V_{1}$ is larger than $\max \left(V_{2}, \max _{j \in[k]} j \cdot v_{j}\right)$ participant $\left(k, V_{1}\right)$ wins. If $V_{1}$ is less than $\max \left(V_{2}, \max _{j \in[k]} j \cdot v_{j}\right)$ then she loses to the imageparticipant $\left(k, V_{2}\right)$ if $\max \left(V_{2}, \max _{j \in[k]} j \cdot v_{j}\right)=V_{2}$, or loses to the text-participants if $\max \left(V_{2}, \max _{j \in[k]} j \cdot v_{j}\right)=\max _{j \in[k]} j$. $v_{j}$. This means that the critical value of the first imageparticipant is $\max \left(V_{2}, \max _{j \in[k]} j \cdot v_{j}\right)$ if she is the winner.

If $C_{\theta}$ is non-empty then the first $j^{*}$ text-participants win. Let $i \in\left[j^{*}\right]$ be an arbitrary winner. First we observe that for any valuation $v_{i}^{\prime}$ greater than or equal to $\max \left(v_{k+1}, \frac{V_{1}}{j^{*}}\right)$, participant $i$ remains as a winner in type profile $\theta^{\prime}=\left(\left(1, v_{i}^{\prime}\right), \theta_{-i}\right)$. This is because for any such change in valuation of participant $i$ number $j^{*}$ remains in set $C_{\theta^{\prime}}$. Moreover, this change does not add any new number $j^{\prime}$ to $C_{\theta^{\prime}}$ such that $j^{\prime}>j^{*}$ because the valuations of the textparticipants with index greater than $j^{*}$ are not changed in $\theta^{\prime}$.

In order to prove that for any valuation $v_{i}^{\prime}$ less than critical value $\max \left(v_{k+1}, \frac{V_{1}}{j^{*}}\right)$, participant $i$ is not a winner we consider two cases: (A) when the critical value is equal to $\frac{V_{1}}{j^{*}}$, and $(\mathrm{B})$ when the critical value is equal to $v_{k+1}$.

Case (A): We prove this case by contradiction. Let $v_{i}^{\prime}$ be a valuation less than $\frac{V_{1}}{j^{*}}$ for which participant $i$ is in the set of winners in type profile $\theta^{\prime}=\left(\left(1, v_{i}^{\prime}\right), \theta_{-i}\right)$. Because $v_{i}^{\prime}$ is less than $\frac{V_{1}}{j^{*}}$, the number of winners which contains participant $i$ cannot be less than or equal to $j^{*}$ in type profile $\theta^{\prime}$. Let $j^{\prime} \in[k]$ which is greater than $j^{*}$ be the number of winners in $\theta^{\prime}$. This means that there are at least $j^{\prime}$ participants whose valuation is larger than $\frac{V_{1}}{j^{\prime}}$ in $\theta^{\prime}$. Note that all the valuations in $\theta$ is the same as $\theta^{\prime}$ except $v_{i}$ which is decreased to $v_{i}^{\prime}$, therefore, there are also at least $j^{\prime}$ participants whose valuation is larger than $\frac{V_{1}}{j^{\prime}}$ in $\theta$ and hence $j^{\prime}$ is in set $C_{\theta}$. This contradicts with the fact that $j^{*}$ is the largest member of $C_{\theta}$.

case (B): In case (B) we have $\max \left(v_{k+1}, \frac{V_{1}}{j^{*}}\right)=v_{k+1}$ which implies that $k \cdot v_{k+1}$ is larger than $V_{1}$ as $j^{*} \in[k]$. Therefore Case (B) can only happen when $j^{*}=k$. Now consider participant $i$ decreases its valuation to value $v_{i}^{\prime}$ that is less than $v_{k+1}$, then it cannot be a winner as there are $k$ other participants whose valuations are more than $v_{i}^{\prime}$ while we have only $k$ items.

The payment function of MITA is set to the critical values of the winners as specified in Lemma 2 which by using Observation 1 and Lemma 1 implies MITA satisfies IC. Moreover, as the payments are always less than the participants' bid IR property of MITA follows. Finally in the following lemma we show that MITA is revenue monotone.

Lemma 3 Let $\theta^{\prime}$ be the type profile obtained by either increasing the valuation of a participant or adding a new participant to the type profile $\theta$, then we have REVENUE(MITA, $\left.\theta^{\prime}\right) \geq \operatorname{REVENUE}($ MitA, $\theta$ ).

Proof. Let $v_{1} \geq v_{2} \geq \ldots$ be the valuations of textparticipants and $V_{1} \geq V_{2} \geq \ldots$ be the valuations of imageparticipants in $\theta$. Similarly let $v_{1}^{\prime} \geq v_{2}^{\prime} \geq \ldots$ be the valuations of text-participants and $V_{1}^{\prime} \geq V_{2}^{\prime} \geq \ldots$ be the valuations of image-participants in $\theta^{\prime}$. Note that for any $i$ we have $v_{i} \leq v_{i}^{\prime}$ and $V_{i} \leq V_{i}^{\prime}$ as we have one more participant or a higher valuation in $\theta^{\prime}$. Let $x$ be the new added participant or the participant which has higher valuation in $\theta^{\prime}$.

We prove this lemma by considering the value of REVEnUE(MITA, $\theta$ ) for the case when text-participants win and the case when an image-participant wins. If an imageparticipant wins then it means that $V_{1}>\max _{j \in[k]} j \cdot v_{j}$ and she pays $\max \left(V_{2}, \max _{j \in[k]} j \cdot v_{j}\right)$ which is the total revenue.

If text-participants win then it means $V_{1} \leq \max _{j \in[k]} j$. $v_{j}$ and there are $j^{*}$ winners where each of them pays $\max \left(v_{k+1}, \frac{V_{1}}{j^{*}}\right)$. If $\max \left(v_{k+1}, \frac{V_{1}}{j^{*}}\right)=\frac{V_{1}}{j^{*}}$, then the total revenue is $V_{1}$. If $\max \left(v_{k+1}, \frac{V_{1}}{j^{*}}\right)=v_{k+1}$, it implies that $k \cdot v_{k+1}$ is larger than $V_{1}$. Remember that $C_{\theta}=\left\{j \in[k] \mid j \cdot v_{j} \geq V_{1}\right\}$ and $j^{*}=\max _{j \in C_{\theta}} j$ therefore $j^{*}=k$ and hence the total payment of the winners is $k \cdot v_{k+1}$.

In summary the total revenue for type profile $\theta$ is the following.

$$
\begin{aligned}
& \operatorname{REVEnue}(\operatorname{MitA}, \theta)= \\
& \begin{cases}\max \left(V_{2}, \max _{j \in[k]} j \cdot v_{j}\right) & V_{1}>\max _{j \in[k]} j \cdot v_{j}(A) \\
\max \left(V_{1}, k \cdot v_{k+1}\right) & V_{1} \leq \max _{j \in[k]} j \cdot v_{j}(B)\end{cases}
\end{aligned}
$$


Similarly the total revenue for type profile $\theta^{\prime}$ is the following.

$$
\begin{aligned}
& \operatorname{ReVenue}\left(\operatorname{Mita}, \theta^{\prime}\right)= \\
& \begin{cases}\max \left(V_{2}^{\prime}, \max _{j \in[k]} j \cdot v_{j}^{\prime}\right) & V_{1}^{\prime}>\max _{j \in[k]} j \cdot v_{j}^{\prime}(A) \\
\max \left(V_{1}^{\prime}, k \cdot v_{k+1}^{\prime}\right) & V_{1}^{\prime} \leq \max _{j \in[k]} j \cdot v_{j}^{\prime}(B)\end{cases}
\end{aligned}
$$

Note that because for any $i$ we have $v_{i} \leq v_{i}^{\prime}$ and $V_{i} \leq V_{i}^{\prime}$ the following inequalities are straight forward.

$$
\begin{aligned}
V_{1} & \leq V_{1}^{\prime} \\
V_{2} & \leq V_{2}^{\prime} \\
\max _{j \in[k]} j \cdot v_{j} & \leq \max _{j \in[k]} j \cdot v_{j}^{\prime} \\
k \cdot v_{k+1} & \leq k \cdot v_{k+1}^{\prime}
\end{aligned}
$$

If both Revenue(mita, $\theta$ ) and Revenue(mita, $\left.\theta^{\prime}\right)$ take their value from Case $(A)$ then the proof of the lemma follows from Equations (2) and (3). Similarly if both REVEnUe(MitA, $\theta$ ) and REVEnUE(MITA, $\theta^{\prime}$ ) take their value from Case $(B)$ then the proof of the lemma follows from Equations (1) and (4).

If ReVenue(Mita, $\theta$ ) takes its value from Case $(A)$ and REVEnue(MitA, $\theta^{\prime}$ ) takes from Case $(B)$ then it means that participant $x$ is a text-participant which causes $\max _{j \in[k]} j \cdot v_{j}^{\prime}$ to be larger than $V_{1}^{\prime}$. The following proves the theorem for this case.

$$
\begin{array}{lr}
\operatorname{REVEnUe}(\operatorname{mita}, \theta) & \\
=\max \left(V_{2}, \max _{j \in[k]} j \cdot v_{j}\right) & \\
<V_{1} & \begin{array}{r}
\text { ReVenue}(\operatorname{MitA}, \theta) \text { takes } \\
\text { its value from Case }(A)
\end{array} \\
=V_{1}^{\prime} & \text { participant } x \text { is a } \\
\leq \max \left(V_{1}^{\prime}, k \cdot v_{k+1}^{\prime}\right) & \text { text-participant } \\
=\operatorname{REVENUE}\left(\operatorname{MiTA}, \theta^{\prime}\right) &
\end{array}
$$

If Revenue(Mita, $\theta$ ) takes its value from Case $(B)$ and REVEnUE(MITA, $\left.\theta^{\prime}\right)$ takes from Case $(A)$ then it means that participant $x$ is an image-participant. The following proves the theorem for this case.

$$
\begin{aligned}
& \text { REVENUE(Mita, } \theta \text { ) } \\
& =\max \left(V_{1}, k \cdot v_{k+1}\right) \\
& \left.<\max _{j \in[k]} j \cdot v_{j} \quad \text { REVEnUE(MITA, } \theta\right) \text { takes } \\
& \text { its value from Case }(B) \text { and } \\
& \text { the fact that } v_{k} \geq v_{k+1} \\
& =\max _{j \in[k]} j \cdot v_{j}^{\prime} \\
& x \text { is an image-participant } \\
& \leq \max \left(V_{2}^{\prime}, \max _{j \in[k]} j \cdot v_{j}^{\prime}\right) \\
& =\operatorname{REVENUE}\left(\operatorname{MITA}, \theta^{\prime}\right)
\end{aligned}
$$

In the above we proved that MITA satisfies IR, IC, and RM. In the following theorem we bound the PORM of MITA and finish this section.

Theorem 4 PoRM(MITA) $\leq \ln k$.
Proof. Let $A$ be the set of winner(s) which realizes the maximum social welfare in type profile $\theta$. If $A$ contains only one image-participant with valuation $V_{1}$ then we also have $V_{1} \geq \max _{j \in[k]} j \cdot v_{j}$. Mechanism MITA also selects an imageparticipant with the same valuation if $V_{1}>\max _{j \in[k]} j \cdot v_{j}$ and hence $\operatorname{PoRM}(\mathrm{MITA})$ is 1 . Otherwise we have $V_{1}=$ $\max _{j \in[k]} j \cdot v_{j}$ where MITA selects a set of text-participants which overall gives social welfare $V_{1}$ and hence again the PoRM(MITA) is 1.

Now we consider the case when $A$ contains textparticipants. By adding enough dummy participants with value zero, and without loss of generality, we assume that set $A$ contains the first $k$ text-participants with highest valuations $v_{1} \geq v_{2} \geq \ldots \geq v_{k}$. Mechanism MITA selects either the first $j^{*}$ text-participants with highest valuations $\left(v_{1} \geq v_{2} \geq \ldots \geq v_{j^{*}}\right)$ or selects an image-participant with valuation $V_{1}$. Remember that $j^{*}$ is the greatest number in set $C_{\theta}=\left\{j \mid j \in[k] \wedge j \cdot v_{j} \geq V_{1}\right\}$ which implies the following.

$$
\forall j^{\prime} \in\left\{j^{*}+1, \ldots, k\right\} \quad v_{j^{\prime}}<\frac{V_{1}}{j^{\prime}}
$$

Note that if MITA selects an image-participant then Equation (5) holds for $j^{*}=0$.

Now we consider the following two cases to prove the theorem.

If MITA selects an image-participant then we have the following.

$$
\begin{aligned}
\operatorname{PoRM}(\text { Mita }) & =\frac{\sum_{j \in[k]} v_{j}}{V_{1}} \\
& \leq \frac{\sum_{j \in[k]} V_{1} / j}{V_{1}} \quad \text { Equation (5) } \\
& \leq \ln k
\end{aligned}
$$

If MITA selects the first $j^{*}$ text-participants then we have the following.

$$
\begin{aligned}
\operatorname{PoRM}(\mathrm{MITA}) & =\frac{\sum_{j \in[k]} v_{j}}{\sum_{j \in\left[j^{*}\right]} v_{j}} \\
& \leq \frac{\sum_{j \in\left[j^{*}\right]} v_{j}+\sum_{j=j^{*}+1}^{k} v_{j}}{\sum_{j \in\left[j^{*}\right]} v_{j}} \\
& \leq \frac{\sum_{j \in\left[j^{*}\right]} v_{j}+\sum_{j=j^{*}+1}^{k} V_{1} / j}{\sum_{j \in\left[j^{*}\right]} v_{j}} \\
& \leq \frac{\sum_{j \in\left[j^{*}\right]} v_{j}+\sum_{j=j^{*}+1}^{k}\left(\sum_{j \in\left[j^{*}\right]} v_{j}\right) / j}{\sum_{j \in\left[j^{*}\right]} v_{j}} \\
& \text { because } V_{1} \leq \sum_{j \in\left[j^{*}\right]} v_{j} \\
& \leq \ln k
\end{aligned}
$$

\section{VIDEO-POD AUCTIONS}

In this section we design a Mechanism for Video-Pod Auction (MVPA) which satisfies IR, IC, and RM whose PoRM is at most $(\lfloor\log k\rfloor+1) \cdot(2+\ln k)$. Note that all the $\log$ functions are in base 2 . Let $\theta=\left(\left(d_{1}, v_{1}\right), \ldots,\left(d_{n}, v_{n}\right)\right) \in \Theta^{n}$ be an arbitrary type profile of $n$ participants. We define the allocation and payment function of MVPA for this type profile. 
Mechanism MVPA partitions the participants into $\lfloor\log k\rfloor+$ 1 groups $G^{(1)}, \ldots, G^{(\lfloor\log k\rfloor+1)}$ where group $G^{(g)}$ contains all the participants whose demand is in the range $\left[2^{g-1}, 2^{g}\right)$. Mechanism MVPA selects winners only from one group $G^{(g)}$.

Definition 5 Let $M^{(g)}$ be equal to $\max \left(\left\lfloor\frac{k}{2^{g}}\right\rfloor, 1\right)$ which is the maximum number of winners MVPA selects from group $G^{(g)}$.

Note that we can select at least $\left\lfloor\frac{k}{2^{g}}\right\rfloor$ winners from $G^{(g)}$ since there are $k$ items and the demand of each participant is at most $2^{g}$. Moreover, from the last group $G^{(\lfloor\log k\rfloor+1)}$ we can select at least one winner although $\left\lfloor\frac{k}{2(\lfloor\log k\rfloor+1)}\right\rfloor=0$, since we assume the demand of all the participants are from the set $[k]$.

Let $\left(d_{1}^{(g)}, v_{1}^{(g)}\right), \ldots,\left(d_{p}^{(g)}, v_{p}^{(g)}\right)$ be the types of all the participants in group $g$ where $p=\left|G^{(g)}\right|$. Here by adding enough dummy participants, we assume $p$ is always larger than $M^{(g)}$. Also, without loss of generality we assume $v_{1}^{(g)} \geq v_{2}^{(g)} \geq \ldots \geq v_{p}^{(g)}$. We define the Max Possible Revenue of Group $g$ (MPRG $(g))$ to be the following.

$$
\operatorname{MPRG}(g)=\max _{j \in\left[M^{(g)}\right]} j \cdot v_{j}^{(g)}
$$

As the name MPRG suggests, we will see that its value captures the maximum revenue can be truthfully obtained from group $g$. Let $G^{\left(g^{*}\right)}$ be a group with the maximum MPRG and $G^{\left(g^{\prime}\right)}$ be a group with the second maximum MPRG breaking the ties arbitrarily.

The set of winners selected by MVPA is

$$
\left\{\left(d_{1}^{\left(g^{*}\right)}, v_{1}^{\left(g^{*}\right)}\right), \ldots,\left(d_{j}^{\left(g^{*}\right)}, v_{j}^{\left(g^{*}\right)}\right)\right\}
$$

where $j$ is the largest number in $\left[M^{\left(g^{*}\right)}\right]$ for which $j \cdot v_{j}^{\left(g^{*}\right)}$ is larger than or equal to $\operatorname{MPRG}\left(g^{\prime}\right)$. In other words, the number of winners $(j)$ is the largest number in $\left[M^{\left(g^{*}\right)}\right]$ for which $j \cdot v_{j}^{\left(g^{*}\right)} \geq \operatorname{MPRG}\left(g^{\prime}\right)$.

Now we use Lemma 1 to show that MVPA is truthful and obtain the payments of winners.

\section{Observation 2 Allocation function $x^{\mathrm{MVPA}}$ is weakly monotone.}

Proof. Note that MVPA sorts the participants according to their valuation and selects the first $j$ participants. Therefore if any participant $i$ increases its valuation it only helps her to enter the winning set. Hence, the observation follows.

In the rest of this section we drop the group identifier of $M^{\left(g^{*}\right)}$ and simply use $M$ unless it is about another group.

In the following lemma we find the critical value of each winner $i$ which is actually equal to its payment ( $\left.p_{i}^{\mathrm{MVPA}}\right)$.

Lemma 4 Let set of winners $x^{\mathrm{MVPA}}(\theta)$ contains the first $j$ participants with highest valuations from $G^{\left(g^{*}\right)}$ and $v_{M+1}^{\left(g^{*}\right)}$ be the $(M+1)$ th highest valuation in group $G^{\left(g^{*}\right)}$ which is zero if it does not exist. Then, the payment of participant $i$ is the following.

$$
p_{i}^{\mathrm{MVPA}}(\theta)= \begin{cases}\max \left(\frac{\mathrm{MPRG}\left(g^{\prime}\right)}{j}, v_{M+1}^{\left(g^{*}\right)}\right) & i \in x^{\mathrm{MVPA}}(\theta) \\ 0 & i \notin x^{\mathrm{MVPA}}(\theta)\end{cases}
$$

Proof. If participant $i$ is not a winner then its payment is zero. When participant $i$ is a winner then we prove that its payment is equal to its critical value (Definition 4 ). In order to prove that value $\max \left(\frac{\operatorname{MPRG}\left(g^{\prime}\right)}{j}, v_{M+1}^{\left(g^{*}\right)}\right)$ is the critical value of participant $i$, we show that for any value larger than $\max \left(\frac{\mathrm{MPRG}\left(g^{\prime}\right)}{j}, v_{M+1}^{\left(g^{*}\right)}\right)$ participant $i$ still wins and for any value less than it she loses.

Remember that $v_{1}^{\left(g^{*}\right)} \geq v_{2}^{\left(g^{*}\right)} \geq \ldots \geq v_{p}^{\left(g^{*}\right)}$ are the valuations of participants in group $G^{\left(g^{*}\right)}$ and $v_{1}^{\left(g^{*}\right)}, v_{2}^{\left(g^{*}\right)}, \ldots, v_{j}^{\left(g^{*}\right)}$ are the valuations of the winners. Because group $G^{\left(g^{*}\right)}$ is the group with the maximum MPRG, we have $v_{j}^{\left(g^{*}\right)} \geq \frac{\operatorname{MPRG}\left(g^{\prime}\right)}{j}$. As there can be at most $M$ winners from group $G^{\left(g^{*}\right)}$ we have $v_{j}^{\left(g^{*}\right)} \geq v_{M+1}^{\left(g^{*}\right)}$. Therefore we have

$$
v_{j}^{\left(g^{*}\right)} \geq \max \left(\frac{\operatorname{MPRG}\left(g^{\prime}\right)}{j}, v_{M+1}^{\left(g^{*}\right)}\right) .
$$

Let participant $i$ with type profile $\left(d_{i}^{\left(g^{*}\right)}, v_{i}^{\left(g^{*}\right)}\right)$ be the $i$ th winner in group $g^{*}$ where $i \in[j]$. We show that for any valuation greater than or equal to $\max \left(\frac{\operatorname{MPRG}\left(g^{\prime}\right)}{j}, v_{M+1}^{\left(g^{*}\right)}\right)$ participant $i$ remains in the winning set. Equation (6) implies that there are $j$ participants in group $G^{\left(g^{*}\right)}$ whose valuations are larger than $\max \left(\frac{\mathrm{MPRG}\left(g^{\prime}\right)}{j}, v_{M+1}^{\left(g^{*}\right)}\right)$. If we decrease the valuation of participant $i$ to $\max \left(\frac{\mathrm{MPRG}\left(g^{\prime}\right)}{j}, v_{M+1}^{\left(g^{*}\right)}\right)$ we still have $j$ participants in group $G^{\left(g^{*}\right)}$ with valuations at least $\max \left(\frac{\operatorname{MPRG}\left(g^{\prime}\right)}{j}, v_{M+1}^{\left(g^{*}\right)}\right)$. Therefore, the value $\operatorname{MPRG}\left(g^{*}\right)$ will be at least MPRG $\left(g^{\prime}\right)$ and group $G^{\left(g^{*}\right)}$ remains the winning group, ' hence participant $i$ remains in the winning set.

Now we prove that if the valuation of participant $i$ is less than the $\max \left(\frac{\operatorname{MPRG}\left(g^{\prime}\right)}{j}, v_{M+1}^{\left(g^{*}\right)}\right)$, she cannot be in the winning set. In order to prove this we consider two cases: (A) when $\max \left(\frac{\operatorname{MPRG}\left(g^{\prime}\right)}{j}, v_{M+1}^{\left(g^{*}\right)}\right)$ is equal to $v_{M+1}^{\left(g^{*}\right)}$, and (B) when $\max \left(\frac{\operatorname{MPRG}\left(g^{\prime}\right)}{j}, v_{M+1}^{\left(g^{*}\right)}\right)$ is equal to $\frac{\operatorname{MPRG}\left(g^{\prime}\right)}{j}$.

Case (A): If $\max \left(\frac{\operatorname{MPRG}\left(g^{\prime}\right)}{j}, v_{M+1}^{\left(g^{*}\right)}\right)=v_{M+1}^{\left(g^{*}\right)}$ and the valuation of participant $i$ is less than $v_{M+1}^{\left(g^{*}\right)}$ then it means that there are $M$ participants who have valuations greater than the valuation of participant $i$. As there can be at most $M$ winners from group $G^{\left(g^{*}\right)}$, participant $i$ cannot be a winner.

Case (B): We prove this case by contradiction. Suppose $\max \left(\frac{\operatorname{MPRG}\left(g^{\prime}\right)}{j}, v_{M+1}^{\left(g^{*}\right)}\right)=\frac{\operatorname{MPRG}\left(g^{\prime}\right)}{j}$ and $\theta^{\prime}=$ $\left(\left(d_{i}^{\left(g^{*}\right)}, v_{i}^{\left(g^{*}\right)^{\prime}}\right), \theta_{-i}\right)$ be a type profile in which the valuation of participant $i$ is less than $\frac{\operatorname{MPRG}\left(g^{\prime}\right)}{j}$ while she is still winner. Because the valuation of participant $i\left(v_{i}^{\left(g^{*}\right)^{\prime}}\right)$ is less than $\frac{\operatorname{MPRG}\left(g^{\prime}\right)}{j}$ and $i$ is in the winning set, in order for $\operatorname{MPRG}\left(g^{*}\right)$ to be larger than $\operatorname{MPRG}\left(g^{\prime}\right)$, there has to be more than $j$ winners . Let $j^{\prime}>j$ be the number of winners in $\theta^{\prime}$. Having $j^{\prime}$ winners in $\theta^{\prime}$ and in order for $G^{\left(g^{*}\right)}$ to be the group with the highest MPRG we conclude that there are $j^{\prime}$ participants with valuation greater than $\frac{\mathrm{MPRG}\left(g^{\prime}\right)}{j^{\prime}}$. Note that the only difference between $\theta$ and $\theta^{\prime}$ is that the valuation of participant $i$ is higher in $\theta$. Therefore, there are also at least $j^{\prime}$ participants with valuation greater than $\frac{\operatorname{MPRG}\left(g^{\prime}\right)}{j^{\prime}}$ in $\theta$. This contradicts with the way we select the number of winners $(j)$ in $\theta$ which is the maximum number for which $j \cdot v_{j}^{\left(g^{*}\right)}$ is larger than MPRG $\left(g^{\prime}\right)$. 
The allocation function $x^{\mathrm{MVPA}}$ is weakly monotone (Observation 2) and the payments of the winners are their critical values (Lemma 4), therefore by Lemma 1 we conclude that MVPA satisfies IC.

In the rest of this section first we prove that MVPA satisfies RM and then bound its PoRM.

Proposition 1 The total revenue of mechanism MVPA for type profile $\theta$ (REVENUE(MVPA, $\theta$ )) is the following.

$$
\operatorname{REVENUE}(\operatorname{MVPA}, \theta)=\max \left(\operatorname{MPRG}\left(g^{\prime}\right), M \cdot v_{M+1}^{\left(g^{*}\right)}\right)
$$

where $g^{\prime}$ is a group with the second highest MPRG.

Proof. From Lemma 4 we know that there are $j$ winners and each of them pay $\max \left(\frac{\operatorname{MPRG}\left(g^{\prime}\right)}{j}, v_{M+1}^{\left(g^{*}\right)}\right)$. Therefore the sum of payments or the revenue of MVPA is $j$. $\max \left(\frac{\operatorname{MPRG}\left(g^{\prime}\right)}{j}, v_{M+1}^{\left(g^{*}\right)}\right)$. The proof of the proposition follows if we show that when $\max \left(\frac{\operatorname{MPRG}\left(g^{\prime}\right)}{j}, v_{M+1}^{\left(g^{*}\right)}\right)$ is equal to $v_{M+1}^{\left(g^{*}\right)}$ then the number of winners $(j)$ is equal to $M$.

If $\max \left(\frac{\operatorname{MPRG}\left(g^{\prime}\right)}{j}, v_{M+1}^{\left(g^{*}\right)}\right)$ is equal to $v_{M+1}^{\left(g^{*}\right)}$ then as $v_{M+1}^{\left(g^{*}\right)} \leq$ $v_{M}^{\left(g^{*}\right)}$, we have $M \cdot v_{M}^{\left(g^{*}\right)} \geq \frac{\operatorname{MPRG}\left(g^{\prime}\right)}{j}$. Remember that $j$ is the maximum number in the set $[M]$ for which $j \cdot v_{j}^{\left(g^{*}\right)}$ is larger than $\operatorname{MPRG}\left(g^{\prime}\right)$. Therefore $j$ is equal to $M$.

Lemma 5 Let $\theta^{\prime}$ be the type profile obtained by either adding a new participant or increasing the valuation of a participant in $\theta$. Then,

$$
\text { REVENUE(MVPA, } \left.\theta^{\prime}\right) \geq \operatorname{REVENUE}(\text { MVPA }, \theta) \text {. }
$$

Proof. Let $x$ be the new added participant or the participant which has the increased valuation in $\theta^{\prime}$. Throughout the proof we show MPRG of each group $g$ in type profile $\theta$ by $\operatorname{MPRG}_{\theta}(g)$ and in type profile $\theta^{\prime}$ by $\operatorname{MPRG}_{\theta^{\prime}}(g)$. Similarly, we show the $j$ th highest valuation of the participants of group $g$ by $v_{j}^{\left(g^{*}, \theta\right)}$ in type profile $\theta$ and by $v_{j}^{\left(g^{*}, \theta^{\prime}\right)}$ in type profile $\theta^{\prime}$.

As the $j$ th highest valuation of the participants of each group can only increase by adding participant $x$, we conclude

$$
\forall g, \forall j \quad v_{j}^{\left(g, \theta^{\prime}\right)} \geq v_{j}^{(g, \theta)} .
$$

Remember that $\mathrm{MPRG}_{\theta}$ of each group $g$ is $\max _{j \in\left[M^{(g)}\right]} j$. $v_{j}^{(g, \theta)}$ and using Equation (7) we get

$$
\forall g \quad \operatorname{MPRG}_{\theta^{\prime}}(g) \geq \operatorname{MPRG}_{\theta}(g) .
$$

In order to prove this lemma we consider two cases: (A) adding participant $x$ does not change the winning group $G^{\left(g^{*}\right)}$, and (B) adding $x$ changes the winning group.

Case (A): Let $g^{\prime \prime}$ be a group with the second highest MPRG in $\theta^{\prime}$, it is possible that $g^{\prime}$ is equal to $g^{\prime \prime}$.

$$
\begin{aligned}
\operatorname{REVEnUE}\left(\text { MVPA }, \theta^{\prime}\right)= & \max \left(\operatorname{MPRG}_{\theta^{\prime}}\left(g^{\prime \prime}\right), M \cdot v_{M+1}^{\left(g^{*}, \theta^{\prime}\right)}\right) \\
& \operatorname{Proposition} 1 \\
\geq & \max \left(\operatorname{MPRG}_{\theta^{\prime}}\left(g^{\prime}\right), M \cdot v_{M+1}^{\left(g^{*}, \theta^{\prime}\right)}\right) \\
& \operatorname{definition~of~} g^{\prime \prime} \\
\geq & \max \left(\operatorname{MrRG}_{\theta}\left(g^{\prime}\right), M \cdot v_{M+1}^{\left(g^{*}, \theta\right)}\right) \\
& \operatorname{Equations}(7) \text { and }(8) \\
= & \operatorname{REVENUE}(\mathrm{MVPA}, \theta)
\end{aligned}
$$

Case (B): Let $G^{\left(g^{\prime \prime}\right)}$ be a group with the highest MPRG in $\theta^{\prime}$. We have

$$
\operatorname{MPRG}_{\theta}\left(g^{*}\right) \geq \operatorname{MPRG}_{\theta}\left(g^{\prime}\right)
$$

as $g^{*}$ has the highest and $g^{\prime}$ has the second highest MPRG in $\theta$.

$$
\begin{aligned}
& \operatorname{MPRG}_{\theta}\left(g^{*}\right) \geq M \cdot v_{M}^{\left(g^{*}, \theta\right)} \quad \operatorname{As}_{\operatorname{MPRG}_{\theta}}\left(g^{*}\right)=\max _{j \in[M]} j \cdot v_{j}^{\left(g^{*}, \theta\right)} \\
& \geq M \cdot v_{M+1}^{\left(g^{*}, \theta\right)} \quad \text { As } v_{M}^{\left(g^{*}, \theta\right)} \geq v_{M+1}^{\left(g^{*}, \theta\right)}
\end{aligned}
$$

Let $\hat{g}$ be the group with second highest MPRG in $\theta^{\prime}$. Because $g^{*}$ is no longer the winning group in $\theta^{\prime}$ it can be a candidate for the group with the second highest MPRG in $\theta^{\prime}$ and hence we have the following.

$$
\operatorname{MPRG}_{\theta}\left(g^{*}\right) \leq \operatorname{MPRG}_{\theta^{\prime}}\left(g^{*}\right) \leq \operatorname{MPRG}_{\theta^{\prime}}(\hat{g})
$$

The following equations conclude the proof of this case.

$$
\begin{aligned}
\operatorname{REVEnUE}(\mathrm{MVPA}, \theta)= & \max \left(\operatorname{MPRG}_{\theta}\left(g^{\prime}\right), M \cdot v_{M+1}^{\left(g^{*}, \theta\right)}\right) \\
\leq & \operatorname{MPRG}_{\theta}\left(g^{*}\right) \\
& \quad \text { by Equations }(9) \text { and }(10) \\
\leq & \operatorname{MPRG}_{\theta^{\prime}}(\hat{g}) \\
& \text { by Equation }(11) \\
\leq & \max \left(\operatorname{MPRG}_{\theta^{\prime}}(\hat{g}), M^{\left(g^{\prime \prime}\right)} \cdot v_{M^{\left(g^{\prime \prime}\right)}+1}^{\left(g^{\prime \prime}, \theta\right)}\right) \\
= & \operatorname{REVEnUE}\left(\operatorname{MVPA}, \theta^{\prime}\right)
\end{aligned}
$$

The following lemma which bounds PoRM of MVPA finishes this section.

\section{Theorem 5 PoRM(MITA $) \leq(\lfloor\log k\rfloor+1) \cdot(2+\ln k)$}

Proof. Let $\operatorname{WF}(g)$ to be the maximum social welfare achievable if we select the winners only from group $G^{(g)}$. Let $A$ be a set of winner(s) which realizes the maximum welfare in type profile $\theta$. Note that as there are $\lfloor\log k\rfloor+1$ groups, one group $(\hat{g})$ has a subset of participants from $A$ whose social welfare is at least $\frac{\mathrm{WF}(A)}{\lfloor\log k\rfloor+1}$ and hence the following.

$$
\mathrm{WF}(\hat{g}) \geq \frac{\mathrm{WF}(A)}{\lfloor\log k\rfloor+1}
$$

Now we prove the following claim about $\operatorname{MPRG}(\hat{g})$.

Claim $1 \operatorname{MPRG}(\hat{g}) \geq \frac{\mathrm{WF}(\hat{g})}{2+\ln k}$

Proof. Let $B$ be the set of participants from group $G^{(\hat{g})}$ which give the maximum social welfare. Because the demands of all the participants of $G^{(\hat{g})}$ is in range $\left[2^{\hat{g}-1}, 2^{\hat{g}}\right)$, size of $B$ is at most $\left\lfloor k / 2^{\hat{g}-1}\right\rfloor$. Remember from Definition 5 that $M^{(\hat{g})}=\max \left(\left\lfloor k / 2^{(\hat{g})}\right\rfloor, 1\right)$ is the maximum number of winners that MVPA potentially selects from group $G^{(\hat{g})}$. Therefore, we have $|B| \leq 2 \cdot M^{(\hat{g})}+1$.

Throughout the proof, we drop the superscript from $M^{(\hat{g})}$ and simply refer to it as $M$.

Let $v_{1} \geq v_{2} \geq \ldots \geq v_{2 \cdot M+1}$ be the valuations of the participants in $B$; if $B$ has less than $2 \cdot M+1$ participants we add enough dummy participants with valuations zero. 
Remember that $\operatorname{MPRG}(\hat{g})=\max _{j \in[M]} j \cdot v_{j}^{(\hat{g})}$ where $M$ is at least 1 (see Definition 5) which implies

$$
v_{i} \leq \frac{\operatorname{MPRG}(\hat{g})}{i} \quad \forall i \in[M]
$$

The following equations conclude the proof of the claim.

$$
\begin{aligned}
\mathrm{WF}(\hat{g}) & =\sum_{i=1}^{2 \cdot M+1} v_{i} \\
& =\sum_{i=1}^{M} v_{i}+\sum_{i=M+1}^{2 \cdot M+1} v_{i} \\
\leq & \sum_{i=1}^{M} v_{i}+\sum_{i=M+1}^{2 \cdot M+1} v_{M} \\
& \text { replacing } v_{i} \text { with } v_{M} \text { for } i>M \\
\leq & \sum_{i=1}^{M} \frac{\operatorname{MPRG}(\hat{g})}{i}+\sum_{i=M+1}^{2 \cdot M+1} \frac{\operatorname{MPRG}(\hat{g})}{M} \\
& \text { by Equation }(13) \\
\leq & (2+\ln k) \operatorname{MPRG}(\hat{g})
\end{aligned}
$$

Remember $G^{\left(g^{*}\right)}$ is the group with maximum MPRG value. Let $j$ be the number for which $\operatorname{MPRG}\left(g^{*}\right)$ is equal to $j \cdot v_{j}^{\left(g^{*}\right)}$. Allocation function $x^{\mathrm{MVPA}}$ selects the first $j^{*}$ participants from group $G^{\left(g^{*}\right)}$ where $j^{*}$ is the maximum number for which $j^{*} \cdot v_{j^{*}}^{\left(g^{*}\right)}$ is larger than MPRG $\left(g^{\prime}\right)$. Therefore we can conclude that $j \leq j^{*}$ and hence

$$
\operatorname{WF}\left(x^{\operatorname{MVPA}}(\theta)\right) \geq \operatorname{MPRG}\left(g^{*}\right) .
$$

The following equations conclude the proof of the theorem.

$$
\begin{aligned}
\operatorname{WF}\left(x^{\operatorname{MVPA}}(\theta)\right) \geq & \operatorname{MPRG}\left(g^{*}\right) \\
& \quad \text { by Equation }(14) \\
\geq & \operatorname{MPRG}(\hat{g}) \\
& G^{\left(g^{*}\right)} \text { has the highest MPRG } \\
\geq & \frac{\mathrm{WF}(\hat{g})}{2+\ln k} \\
& \operatorname{Claim~} 1 \\
\geq & \frac{\mathrm{WF}(A)}{(\lfloor\log k\rfloor+1) \cdot(2+\ln k)}
\end{aligned}
$$

by Equation (12)

\section{LOWER BOUND}

In this section we prove Theorem 2. As mentioned earlier we need two additional mild assumptions of anonymity and independence of irrelevant alternatives (which we define below) on the class of mechanisms for which we prove our lower bound.

Definition 6 A mechanism $(\mathcal{M}=(x, p))$ is anonymous $(A M)$ if the following holds: Suppose $\theta_{1}, \theta_{2} \in \Theta^{n}$ are two type profiles which are permutations of each other (i.e. the set of type profiles are same just that the identities of participants to whom those types belongs are different). Say $\theta_{2}=\pi\left(\theta_{1}\right)$. Also say $x\left(\theta_{1}\right)=S_{1}$ and $x\left(\theta_{2}\right)=S_{2}$. Then $S_{2}=\pi\left(S_{1}\right)$.
Definition 7 Let $\theta \in \Theta^{n}$ be an arbitrary type profile and $i \in N$ be an arbitrary participant with type $\theta_{i}=\left(d_{i}, v_{i}\right)$. A mechanism $(\mathcal{M}=(x, p))$ satisfies Independence of Irrelevant Alternatives (IIA) if we decrease the bid of a losing participant, say participant $i$, to $\hat{v}_{i}<v_{i}$ then the new set of winners is a super set of the previous one, i.e., $x(\theta) \subseteq x\left(\left(d_{i}, \hat{v}_{i}\right), \theta_{-i}\right)$. In other words, decreasing the bid of a losing participant does not hurt any winner.

The proof outline of Theorem 2 is the following. Let $\mathcal{M}^{*}=\left(x^{*}, p^{*}\right)$ be a mechanism which satisfies all the five properties and has the optimal PoRM OPT (i.e., OPT $=$ $\left.\operatorname{PoRM}\left(\mathcal{M}^{*}\right)\right)$. We study the behavior of $\mathcal{M}^{*}$ in a few type profiles. Let $\epsilon$ be an arbitrary small positive real value. First we show that when there are only two participants with types $(k, 1)$ and $(k, 1+\epsilon), \mathcal{M}^{*}$ gives all the $k$ items to the participant with type $(k, 1+\epsilon)$. The revenue of $\mathcal{M}^{*}$ from these two participants is 1 . Then, we add $k$ more participants to create type profile $\theta=$ $\left((1,1-\epsilon),\left(1, \frac{1}{2}-\epsilon\right), \ldots,\left(1, \frac{1}{k}-\epsilon\right),(k, 1),(k, 1+\epsilon)\right) . \quad$ The $\mathrm{RM}$ property requires $\mathcal{M}^{*}$ to make at least the same revenue for $\theta$. From this constraint we are able to show that $\mathcal{M}^{*}$ assigns all the items to participant $k+2$ with type $(k, 1+\epsilon)$ and hence gets social welfare $1+\epsilon$. Note that the maximum social welfare happens when the set of winners is $\{1, \ldots, k\}$ which implies $\operatorname{WFR}\left(\mathcal{M}^{*}, \theta\right) \geq \sum_{i=1}^{k} \frac{1}{i}-k \cdot \epsilon$ (see Definition 1). Because $\operatorname{PoRM}\left(\mathcal{M}^{*}\right) \geq \operatorname{wFR}\left(\mathcal{M}^{*}, \theta\right)$ for any $\theta \in \Theta^{n}$ we conclude that OPT $\geq \sum_{i=1}^{k} \frac{1}{i}$.

First we study the behavior of $\mathcal{M}^{*}$ when we have only two participants with types $(k, 1)$ and $(k, 1+\epsilon)$.

Lemma 6 Mechanism $\mathcal{M}^{*}$ in type profile $((k, 1),(k, 1+\epsilon))$ gives all $k$ items to the second participant and make one unit of revenue, i.e., $x^{*}((k, 1),(k, 1+\epsilon))=\{2\}$ and $p^{*}((k, 1),(k, 1+\epsilon))=(0,1)$.

Proof. First we study type profile $\left(\left(k, v_{1}\right),\left(k, v_{2}\right)\right)$ for general values $v_{1}, v_{2} \in \mathbb{R}^{+}$where $v_{1}<v_{2}$. We prove that $\mathcal{M}^{*}$ gives all the items to the second participant.

Claim $2 x^{*}\left(\left(k, v_{1}\right),\left(k, v_{2}\right)\right)=\{2\}$ for any $v_{1}, v_{2} \in \mathbb{R}^{+}$ where $v_{1}<v_{2}$.

Proof. First note that $M^{*}$ has to have a winner for this type profile because otherwise its social welfare will be zero while the maximum social welfare is $v_{2}$. This makes the social welfare ratio of $\mathcal{M}^{*}$ to be undefined.

Now we prove that if $x^{*}\left(\left(k, v_{1}\right),\left(k, v_{2}\right)\right)=\{1\}$ then $\mathcal{M}^{*}$ either violates IC or AM. Lets call type profile $\left(\left(k, v_{1}\right),\left(k, v_{2}\right)\right)$ by $\theta^{(1)}$ and suppose for the sake of contradiction $x^{*}\left(\theta^{(1)}\right)=$ $\{1\}$. From Lemma 1 we know that if participant 1 increases his bid to $v_{2}$ she still wins, hence $x^{*}\left(\theta^{(2)}\right)=\{1\}$ where $\theta^{(2)}=\left(\left(k, v_{2}\right),\left(k, v_{2}\right)\right)$. Now if in type profile $\theta^{(2)}$ participant 2 decrease his bid to $v_{1}$, again from Lemma 1 we conclude that she cannot win, i.e., $x^{*}\left(\theta^{(3)}\right)=\{1\}$ where $\theta^{(3)}=\left(\left(k, v_{2}\right),\left(k, v_{1}\right)\right)$. Type profile $\theta^{(1)}$ is $\theta^{(3)}$ with participant 1 swapped with participant 2 but in both of them the first participant wins which contradicts with AM.

Claim 2 directly proves that the winner in type profile $((k, 1),(k, 1+\epsilon))$ is the second participant. The only thing remains is to show that her payment $\left(p_{2}\right)$ is 1 . Note that payment $p_{2}$ cannot be less than one because otherwise by Lemma 1 participant 2 wins all the items in type profile 
$\left((k, 1),\left(k, p_{2}\right)\right)$ which contradicts with Claim 2. Payment $p_{2}$ cannot be larger than one because otherwise for any value $1<v_{2}<p_{2}$ participant 2 wins all the items in type profile $\left((k, 1),\left(k, v_{2}\right)\right)$. This contradicts with Lemma 1 which states that the payment $p_{2}$ is the smallest value for which participant 2 wins the items.

Now we add $k$ more participants each of which wants only one item. In the following lemma we prove that RM forces $\mathcal{M}^{*}$ to assign all of the items to one of the participants who want all the items.

Lemma 7 For the set of $k+2$ participants with type profile $\theta^{(0)}=\left((1,1-\epsilon),\left(1, \frac{1}{2}-\epsilon\right), \ldots,\left(1, \frac{1}{k}-\epsilon\right),(k, 1),(k, 1+\epsilon)\right)$, mechanism $\mathcal{M}^{*}$ assigns all the $k$ items to either participant $k+1$ or participant $k+2$, i.e., $x^{*}\left(\theta^{(0)}\right)=\{k+1\}$ or $x^{*}\left(\theta^{(0)}\right)=\{k+2\}$.

Proof. We prove the lemma by contradiction that if $\mathcal{M}^{*}$ assigns the items to a subset of the first $k$ participants it satisfy be RM. We consider a class of $k$ type profiles $\left(\theta^{(1)}, \ldots, \theta^{(k)}\right)$ where $\theta^{(i)}$ is built from $\theta^{(i-1)}$. The only possible difference between $\theta^{(i)}$ and $\theta^{(i-1)}$ is in the valuation of participant $i$. If participant $i$ is a winner in $\theta^{(i-1)}$, then we obtain $\theta^{(i)}$ by increasing the valuation of the $i$ th participant from $\frac{1}{i}-\epsilon$ to $1-\epsilon$. Note that the payment of participant $i$ in $\theta^{(i-1)}$ is at most her valuation which is $\frac{1}{i}-\epsilon$ and in $\theta^{(i)}$ it remains the same by Lemma 1 . If participant $i$ is not a winner in $\theta^{(i-1)}$ then we obtain $\theta^{(i)}$ by decreasing his valuation to zero. Note that by IIA, no winner turns to a loser in $\theta^{(i)}$.

Let $j \in\{1, \ldots, k\}$ be the largest number for which participant $j$ is a winner in $\theta^{(j-1)}$ and we increase his valuation to $1-\epsilon$ in $\theta^{(j)}$. Note that at the start in type profile $\theta^{(0)}$ the set of winners is a non-empty subset of $\{1, \ldots, k\}$. Therefore there is at least one such $j$ for which participant $j$ is a winner in $\theta^{(j)}$ since decreasing the non-winners valuation does not reduce the size of the winners.

Now we prove that there is no winner in the set of participants $\{j+1, \ldots, k\}$ in type profile $\theta^{(j)}$. Assume otherwise and let $p \in\{j+1, \ldots, k\}$ be the smallest number for which participant $p$ is a winner in $\theta^{(j)}$. Note that when we decrease the valuation of each participant $j<p^{\prime}<p$ to zero to obtain $\theta^{\left(p^{\prime}\right)}$, participant $p$ remains as a winner in all of them by IIA. Therefore, participant $p$ is a winner in type profile $\theta^{(p-1)}$ and we increase his valuation in $\theta^{(p)}$ which contradicts with the fact that $j$ is the largest number for which participant $j$ is a winner in $\theta^{(j-1)}$.
The payment of participant $j$ in $\theta^{(j-1)}$ is at most its valuation which is $\frac{1}{j}-\epsilon$. When we increase his bid to $1-\epsilon$ in type profile $\theta^{(j)}$ its payment remains the same by Lemma 1 . Note that by construction of $\theta^{(j)}$ the valuation of all participants in $\{1, \ldots, j\}$ is either zero or $1-\epsilon$. If the valuation of them is $1-\epsilon$ and they are winner, by AM their payment is $\frac{1}{j}-\epsilon$. Therefore the total payments or revenue of $\mathcal{M}^{*}$ in $\theta^{(j)}$ is at most $j \cdot\left(\frac{1}{j}-\epsilon\right)=1-j \cdot \epsilon$ since there is no other winner in set of participants $\{j+1, \ldots, k\}$ in type profile $\theta^{(j)}$.

Note that type profile $\theta^{(j)}$ is obtained from type profile $((k, 1),(k, 1+\epsilon))$ by adding $k$ more participants. However the revenue of $\theta^{(j)}$ is $1-j \cdot \epsilon$ that is strictly less than 1 which is the revenue of $((k, 1),(k, 1+\epsilon))$ by Lemma 6 . This contradicts with the RM property of $\mathcal{M}^{*}$, hence $\mathcal{M}^{*}$ has to assign the items to either participant $k+1$ or $k+2$.

Now we show how from Lemma 7 we can derive Theorem 2. Note that the maximum welfare for type profile $\theta^{(0)}=\left((1,1-\epsilon),\left(1, \frac{1}{2}-\epsilon\right), \ldots,\left(1, \frac{1}{k}-\epsilon\right),(k, 1),(k, 1+\epsilon)\right)$ realized when we give one item to each of the first $k$ participants for which we get the total social welfare $\sum_{i=1}^{k} \frac{1}{i}-k \cdot \epsilon$, i.e., the nominator of Definition 1 for this type profile is $\sum_{i=1}^{k} \frac{1}{i}-k \cdot \epsilon$. The denominator of Definition 1 is at most $1+\epsilon$ by Lemma 7 . Therefore the ratio of the welfare for this type profile is at least $\frac{\sum_{i=1}^{k} 1 / i-k \cdot \epsilon}{1+\epsilon}$. Because OPT is the maximum ratio over all type profiles (see Definition 2) we have OPT $\geq \frac{\sum_{i=1}^{k} 1 / i-k \cdot \epsilon}{1+\epsilon}$ which results in OPT $\geq \sum_{i=1}^{k} \frac{1}{i}-\epsilon^{\prime}$ where $\epsilon^{\prime}=\frac{\epsilon\left(k-\sum_{i=1}^{k} 1 / i\right)}{1+\epsilon}$.

Note that the value $\epsilon^{\prime}$ can be made arbitrarily small by selecting a sufficiently small value for $\epsilon$. Therefore we prove that for any positive small real value $\epsilon^{\prime}$ we have OPT $\geq$ $\sum_{i=1}^{k} \frac{1}{i}-\epsilon^{\prime}$ which implies Theorem 2 .

\section{ACKNOWLEDGEMENT}

The first author would like to thank Vasilis Gkatzelis for some initial fruitful discussions on this topic. 


\section{REFERENCES}

[1] L. M. Ausubel and P. Milgrom. Ascending auctions with package bidding. Frontiers of Theoretical Economics, 1(1):1-42, 2002.

[2] S. Bikhchandani, S. Chatterji, R. Lavi, A. Mu'alem, N. Nisan, and A. Sen. Weak monotonicity characterizes deterministic dominant-strategy implementation. Econometrica, 74(4):1109-1132, 2006.

[3] R. Day and P. Milgrom. Core-selecting package auctions. International Journal of game Theory, 36(3-4):393-407, 2008.

[4] A. V. Goldberg, J. D. Hartline, A. R. Karlin, M. Saks, and A. Wright. Competitive auctions. Games and Economic Behavior, 55(2):242-269, 2006.

[5] A. V. Goldberg, J. D. Hartline, and A. Wright. Competitive auctions and digital goods. In Symposium on Discrete Algorithms, pages 735-744, 2001.

[6] R. Lavi, A. Mu'Alem, and N. Nisan. Towards a characterization of truthful combinatorial auctions. In Foundations of Computer Science, pages 574-583, 2003.
[7] R. B. Myerson. Optimal auction design. Mathematics of Operations Research, 6(1):58-73, 1981.

[8] N. Nisan, T. Roughgarden, E. Tardos, and V. V. Vazirani. Algorithmic game theory. Cambridge University Press, 2007.

[9] B. Rastegari, A. Condon, and K. Leyton-Brown. Stepwise randomized combinatorial auctions achieve revenue monotonicity. In Symposium on Discrete Algorithms, pages 738-747, 2009.

[10] B. Rastegari, A. Condon, and K. Leyton-Brown. Revenue monotonicity in deterministic, dominant-strategy combinatorial auctions. Artificial Intelligence, 175(2):441-456, 2011.

[11] K. Roberts. The characterization of implementable choice rules. Aggregation and Revelation of Preferences, 12(2):321-348, 1979.

[12] J.-C. Rochet. A necessary and sufficient condition for rationalizability in a quasi-linear context. Journal of Mathematical Economics, 16(2):191-200, 1987.

[13] M. Saks and L. Yu. Weak monotonicity suffices for truthfulness on convex domains. In Electronic Commerce, pages 286-293, 2005. 